\title{
Three-dimensional deformation mapping of Mode I interlaminar crack extension in particle-toughened interlayers \\ G. Borstnar ${ }^{\mathrm{a}}$, F. Gillard ${ }^{\mathrm{b}}$, M.N. Mavrogordato ${ }^{\mathrm{a}}$, I. Sinclair ${ }^{\mathrm{a}}$, S.M. Spearing ${ }^{\mathrm{a}}$ \\ ${ }^{a}$ Engineering Materials, Faculty of Engineering and the Environment, University of Southampton, Southampton SO17 1BJ, UK \\ ${ }^{\mathrm{b}}$ Bioengineering Group, Faculty of Engineering and the Environment, University of Southampton, Southampton SO17 1BJ, UK \\ *Corresponding Author: gb6g09@soton.ac.uk
}

\begin{abstract}
This paper presents the first use of Digital Volume Correlation (DVC) on Carbon Fibre Reinforced Plastics (CFRPs) to quantify the strain fields ahead of a Mode I delamination. DVC is a relatively novel tool that can be used to measure displacements and strains occurring inside materials under load. In conjunction with Computed Tomography (CT), the technique has been applied to porous materials, with results providing strain data for validation of Finite Element (FE) models. However, the application of the technique to laminated materials has been limited, with studies often requiring fiducial markings required for volume correlation. In this work, crack propagation steps were captured at a $325 \mathrm{~nm}$ voxel resolution using Synchrotron Radiation Computed Tomography (SRCT). The material systems investigated featured different crack bridging mechanisms such as; particle-bridges, resin ligaments, and fibre-bridges. An assessment of noise and sub-volume size on the strain measurement determined that the optimal sub-volume size was 150 voxels with $50 \%$ overlap. This provided a spatial resolution of $48.8 \mu \mathrm{m}$ for strain and a corresponding strain resolution ranging between $220-690 \mu \varepsilon$ for the repeated reference scans. A rigid body translation study confirmed that specimen movements perpendicular to the fibre orientation support the 'real' physical displacements. However, along the fibre direction, the correlation was poor, with correct displacements being detected only within the particle-toughened interlayers. The study demonstrates that strain measurements can be made perpendicular to the fibre direction across the interlayer, which could be used to validate future FE models of these poorly understood particle-toughened interlayers.
\end{abstract}

Keywords: Synchrotron radiation computed tomography, Polymer matrix composites, Delamination, Digital Volume Correlation, Local strain distribution 


\section{1 - Introduction}

Carbon Fibre Reinforced Plastics (CFRPs) are increasingly used in primary aerospace structures due to their desirable strength- and stiffness-to-weight properties. However, low velocity impacts that may occur in service can cause significant loss in mechanical properties without producing easily identifiable surface damage [1]. The dispersion of secondary-phase particles within interlaminar regions has been developed to suppress the spread of sub-surface delaminations caused by impacts via toughening of the interlayer [2], [3], [4]. Micromechanical understanding of delamination processes in these complex particletoughened interlayers is not well established, with corresponding uncertainty in where added toughness arises. Through the use of Synchrotron Radiation Computed Tomography (SRCT), in situ non-destructive identification of micro-mechanisms is possible within the bulk material, maintaining mechanical constraint conditions and allowing time-series experiments to capture crack growth under controlled loading conditions [5] [6]. With such data, the DVC technique can be used to quantify the displacements and strains inside the material between the load steps.

Traditionally, strains can be measured using strain gauging, Digital Image Correlation (DIC), shearography, speckle interferometry, Thermal Stress Analysis (TSA) and reflectrometry. However, these techniques can only provide surface measurements of strains at modest spatial resolutions [7]. Internal strains have been measured with diffraction approaches (X-ray and Neutron methods) [8] [9], but are complicated especially when handling non-linear or yielding processes. Through the use of CT, and associated in situ experimental approaches, it is possible to capture this form of damage progression in sufficient detail to apply the DVC technique. Image correlation of volumetric datasets was first implemented to determine continuum-level displacement and strain fields in trabecular bone [10], [11] and helped validate FE models [7], [13]. With CT resolutions now routinely reaching below the micro-meter range, 3D volumes of internal micro-architecture can now be generated with ultra-high resolution micro-focus CT systems [14], [15] and via SRCT [6], [16]. DVC uses the naturally occurring texture in the material to track $3 \mathrm{D}$ displacements of a small sub-volume of interest between two load steps, which can be translated into local strain measurements [11]. Due to the relatively recent uptake of the technique, further investigation is required into the validity of the displacement data produced since it relies heavily on the specimen's internal architecture and CT results (repeatability of contrast-to-noise, spatial resolution, and imaging artefacts). Previous DVC studies have investigated materials such as 
bone [17], rock [18], synthetic foams [19], wood [20] and sand [18], which all offer high contrast in volumetric datasets. Less contrasted materials, such as laminates, have not been explored with DVC. The only known work that used DVC to assess strains within a laminate required fiducial markings, in the form of copper particles, to track displacements during a three-point bend test [13].

In the present study, Mode I delaminations are studied in particle-toughened CFRP laminates with the aim to (a), quantify the strain generation at and ahead of a Mode I delamination tip, which can be seen as the most critical loading condition [21], and (b), to assess the accuracy and validity of the DVC technique. Since this is the first use of the technique to investigate interlaminar strains using SRCT data at such high resolutions, the first section of the paper investigates the strain resolution and the optimal sub-volume parameters via a noise study. This experiment was conducted using stationary and predetermined physical translations of the specimen in order to provide an assessment of the scan repeatability and interpolation errors from the correlation algorithm. The strain resolution is defined as the minimum strain value that can be extracted from the data and regarded as a result of material behaviour and not due to noise in the data or systematic artefacts due to the imaging or signal processing [7]. These known displacements were also used to validate the displacement data from the DVC analysis. The second section of the paper identifies the strain behaviour at the crack tip, with the three different particletoughened systems displaying dissimilar crack bridging mechanisms. Crack propagation in these complex microstructures is highly discontinuous, with no clear 'crack tip' [22]. However, for the first time, interlayer-scale strain measurements have been made that can be compared to micromechanical FE models. The work is intended to support material development and increase the understanding of the fundamental aspects of comparable particle-toughened interlayers that are widely used in modern composite aircraft structures.

\section{2 - Methods}

\section{1 - Materials}

The developmental CFRPs were prepared by Cytec Industries and manufactured and cured according to a standard aerospace autoclave cycle. A uni-directional 16 ply layup $(\sim 3$ mm thick) was prepared from pre-preg, with a $40 \mu \mathrm{m}$ thick Polytetraflouroethylene (PTFE) insert at the mid-plane along one edge of the panel to control the initiation of fracture. The primary reinforcement was a proprietary intermediate modulus carbon fibre $(\sim 5.4 \mu \mathrm{m}$ in 
diameter). The secondary-phase thermoplastic toughening particles were confined to a $\sim 30$ $\mu \mathrm{m}$ thick interlayer present at each ply interface. Three different particle systems are presented in this work, with the fibre type, sizing, base resin and particle volume fraction (13 \%) being consistent between the systems. Material A (Mat. A) is identifiable by its irregularly shaped particles that are 5-20 $\mu \mathrm{m}$ in diameter. Material B (Mat. B) can be identified from a more regular spherical geometry, with particle sizes between 5-10 $\mu \mathrm{m}$. Material C (Mat. C) is a hybrid system, containing both particles featured in Mat A, and another particle type that could not be resolved in the CT data and is suspected to have a high interface strength. The composition of these systems is proprietary and is not important to the key findings of this paper.

\section{2 - Specimen Geometry and Loading}

In order to provide a uniform X-ray path through all angles of rotation and to maximise the transmission of low energy X-ray photons, a cross-sectional geometry of $2.5 \mathrm{x}$ $3 \mathrm{~mm}$ was chosen. The specimens were $150 \mathrm{~mm}$ long with a $10 \mathrm{~mm}$ long PTFE insert. A purpose built screw-driven (displacement controlled) in situ compression rig was used to drive a wedge into the mid-plane of the specimen at the PTFE insert (as seen in Figure 1). A square ram ensured that there was no torsional loading on the specimen. An initial loading step was conducted to grow the crack about $\sim 5 \mathrm{~mm}$ prior to conducting the time-series experiments. Following this, the wedge was driven into the crack to achieve a further $200 \mu \mathrm{m}$ of Mode I crack extension and scanned again in order to capture the crack growth in 3D.

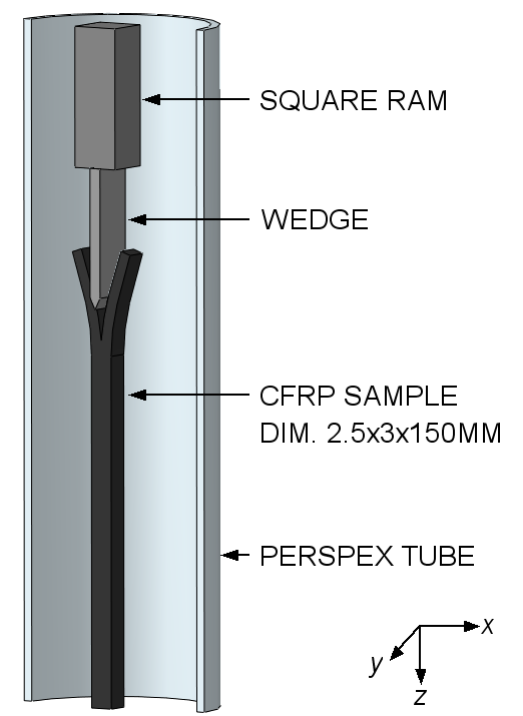

Figure 1 - Schematic of the Mode I wedge-driven loading rig 


\section{2 - Synchrotron Radiation Computed Tomography}

SRCT measurements were carried out on the TOMCAT beamline at the Swiss Light Source, Paul Scherrer Institut, Switzerland. A detector size of 2560 x 2160 pixels was used and scans were conducted at a beam energy of $14 \mathrm{keV}$ and a voxel size of $0.325 \mu \mathrm{m}$. The projection data for each scan consisted of 1601 projections with an exposure of $100 \mathrm{~ms}$ for each projection. After reconstructing using the in-house GRIDREC method [23], the bit depth of the volumes was reduced from a 16-bit to 8-bit format in order to achieve faster processing times for the DVC analysis.

\section{3 - Digital Volume Correlation}

Digital Volume Correlation was performed using DaVis 8.1.3 software [24] via a proprietary Fast Fourier Transform (FFT) approach and a 50\% overlap between neighbouring sub-volumes. Figure 2(a) shows the volume correlation process of two neighbouring subvolumes ' $\mathrm{A}$ ' and 'B', where following a load step, the new position of each sub-volume centroid is determined. The correlation coefficient measures the similarity in the distribution of grey-levels between the original and displaced sub-volume, with the position of the subvolume determined where the correlation is closest to 1 . Similar to DIC, the correlation coefficient can be used to determine how well two volumes are related. A coefficient of 1 means the volume is completely correlated, whereas a value of 0 means they are completely unrelated [7]. This correlation coefficient was used in Section 4 to remove data points where the presence of new crack surfaces within the sub-volumes reduced the accuracy and confidence with which the sub-volume location was determined. The displacement vector fields obtained from the correlation were exported into MATLAB ${ }^{\circledR}[25]$ and the strain was computed using the gradient function (described in [7]). This computed the average strain between the neighbouring sub-volume centroids over the gauge length that was $50 \%$ of the sub-volume size. It should be noted that this measurement was not independent, and that the spatial resolution for strain was equal to the sub-volume size because some grey-scale information is shared in the overlapped volume [7].

Figure 2 (b) shows the raw vertical displacements (crack opening) of sub-volumes at different positions along the ply, depicting the movement of the interlayer and, for clarity, only one side of the crack flank. The relative movement between the sub-volumes in the centre and edge of the interlayer illustrate that the interlayer deformation is contributing most to the total ply opening. Therefore, when interpreting a strain measurement across a gauge 
length containing both interlayer and ply, it should be noted that the dominant source of the sub-volume displacement is determined by the deformation of the interlayer (resin). The approximate crack 'tip' location is shown in Figure 2(b), with the drop in correlation on the left hand side showing the erroneous sub-volume displacements caused by the presence of new crack surfaces. The technique cannot track new features that appear in the sub-volume, but this can still be useful in identifying cracks, as the correlation coefficient will be low. In the results presented, only interlayer strains to the right of this line were considered.

In order to assess the metrological performance of the DVC technique, stationary and rigid body translation studies were conducted prior to deforming the specimen (Section 3 ). Following the identification of the strain resolution and optimal sub-volume parameters, a multi-step approach was implemented during correlation. This maximised the ability for the particles within the interlayers to be used as tracking markers along the fibre-direction, since the larger sub-volumes captured larger displacements and aided placement of the smaller subvolumes. More information on the DVC technique can be found at [10].

(a)

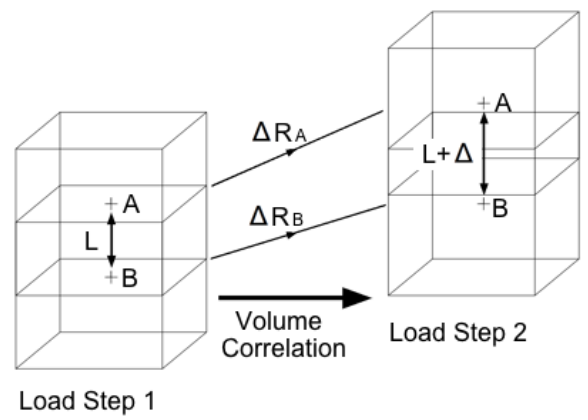

(b)

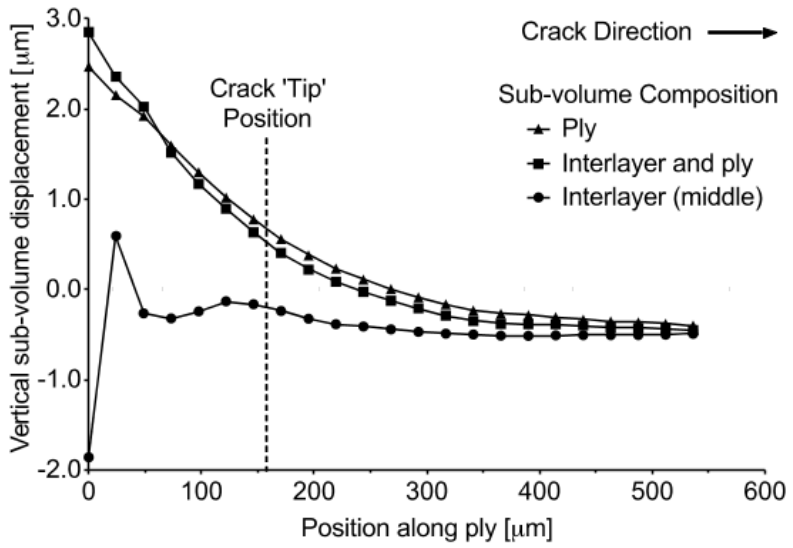

Figure 2 - Illustration showing (a) the correlation of sub-volumes between two load steps and (b) the vertical (crack opening) displacements of sub-volumes along the interlayer and upper crack flank 


\section{3 - Evaluation of strain measurements and limitations}

The noise study involves two analyses. The first is the stationary analysis, which involves repeated scanning of the same specimen without altering the load (zero strain) or scanning parameters (propagation distance, beam energy, exposure, or number of projections). This test aims to quantify the scan noise and/or mechanical inaccuracies. The second test is the rigid body translation, in which the sample was moved by defined displacements both perpendicular to the fibre-direction and parallel to the fibres. Scans were conducted between each movement to assess whether the magnitude and direction of the displacement vector fields are accurate, and to assess the interpolation errors associated with the correlation algorithm.

\section{1 - Stationary Results}

Under ideal conditions, the obtained displacement vector data should be equal to zero. However, noise from the imaging system will mean that this is never the case, and small subvolume displacements will induce false strains that are captured within the results. A conservative way of assessing this error is to take the maximum standard deviation of the strain map [7], [26]. However, this value may be disproportionately affected by local scanning artefacts, such as ring artefacts. In this work, the strain error (referred to as the strain resolution) was calculated by taking the standard deviation of all of the measured strain components within the volume, which form a normal distribution with a mean of $\approx$ zero. This captures the stochastic noise in the imaging system and biases in the correlation algorithm that are also influenced by the material's internal contrast and structure.

A factor that can significantly compromise strain resolution is the sub-volume size used for analysis. Figure 3 shows the influence of changing the sub-volume size on the strain resolution, illustrating the compromise between the strain and spatial resolution, as previously reported in [27] and [28]. An excessively small sub-volume will contain too few features for accurate correlation, which may compromise the strain resolution due to the lack of structure and increased susceptibility to noise. On the other hand, a larger sub-volume will compromise the spatial resolution, since the underlying deformation within will not be captured because the final displacement of the sub-volume is calculated by the average displacement of all the voxels contained within. Figure 4 shows the Mat. A. microstructure in relation to two sub-volume sizes. Therefore, although Figure 3 shows that a larger subvolume produces the best strain resolution, it would provide insufficient spatial resolution to 
capture the interlayer strains. Finally, a sub-volume size of 150 voxels $(48.8 \mu \mathrm{m})$ was chosen since this would provide nominally two strain measurements across the interlayer (due to the $50 \%$ overlap of sub-volumes) and an adequate strain resolution.

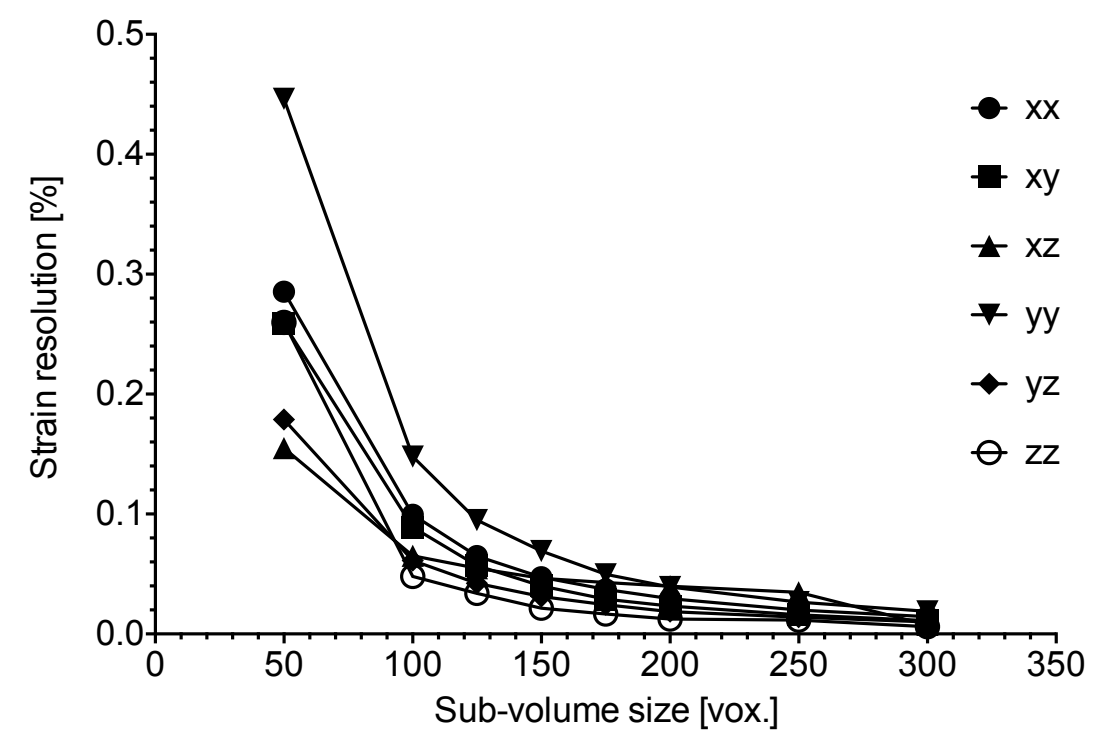

Figure 3 - Influence of the sub-volume size on the strain resolution, illustrating the compromise between strain and spatial resolution

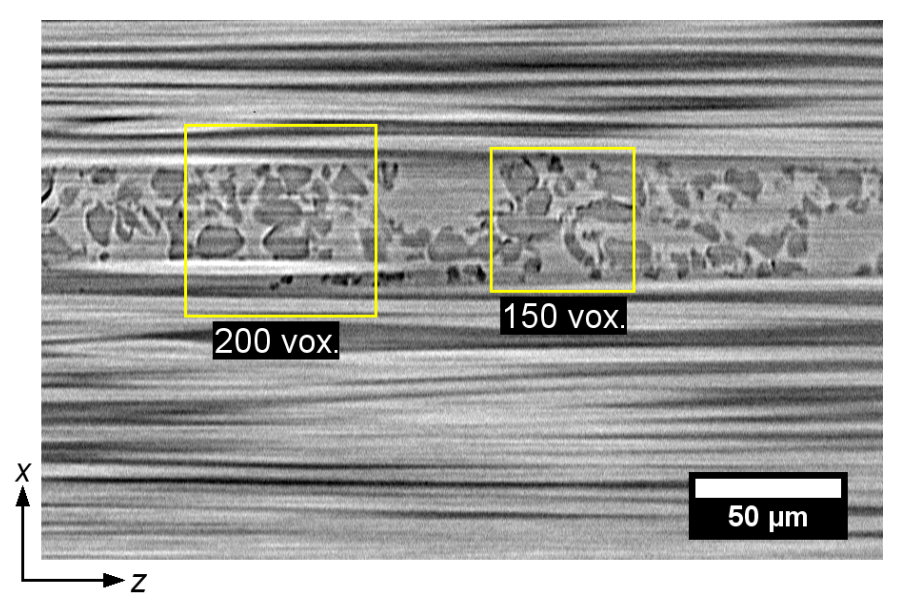

Figure 4 - Illustration of the sub-volume size in relation to the material microstructure, highlighting the anisotropic structure in the fibre bed 


\section{2 - Rigid Body Translation}

Figure 5 (a) and (b) illustrate the displacement fields following a movement across the fibres and along the fibre direction respectively using a sub-volume size of 150 voxels with $50 \%$ overlap and two processing passes. The induced movement across the fibres (ydirection) was manually measured to the nearest pixel in Image $^{\mathrm{TM}}$ to be $16.9 \pm 0.325 \mu \mathrm{m}$. Figure 5(a) indicates displacements ranging between $17.1-17.3 \mu \mathrm{m}$, which is within the accuracy of the manual measurements. The slight gradient across the figure may be due to the 'top-heavy' loading rig tipping over and rotating slightly following the movement away from the centre of the rotation stage. However, in absolute terms, the variation is small (less than a voxel). The measured vertical movement was $1.3 \pm 0.325 \mu \mathrm{m}$, but the displacements obtained via DVC (Figure 5(b)) were only accurate within the interlayers, where a vertical displacement of about $1.4 \mu \mathrm{m}$ was recorded. This result elucidates a known limitation of the DVC technique, in which the correlation along the fibre direction is difficult because individual features are less unique to a particular sub-volume [20]. The cylindrical structure makes finding a correlation peak along the anisotropic architecture difficult due to the presence of many local correlation peaks along the fibres, meaning that the displacement is not accurately identified. This is illustrated in Figure 4, where there is a less unique pattern in the fibre bed along the fibre direction. This problem has been tackled by using fiducial markings in such architectures to allow the DVC to track displacements; however, the inclusion of, for example $150 \mu \mathrm{m}$ metallic particles (used in [13]), will undoubtedly disturb the microstructure investigated here. Nonetheless, the magnitude and direction of the movements perpendicular to the fibres is within the manual measurements, which allows strain measurements across the fibres to be taken with confidence provided that the volumes are aligned in the fibre direction as a precaution prior to analysis. Also, rigid body motion along the fibres is not expected to occur due to the in situ configuration of the experiment.

Interestingly, there is enough contrast between the particles and resin to allow displacement tracking along the fibre-direction. The gradient of z-displacements occurring between the interlayers in Figure 5(b) is probably due to the multi-step approach finding the displacement of the larger 300 vox. sub-volumes first, which are then used to aid the search for the correlation peak of the smaller sub-volumes. Given that the samples during the rigid body translation tests were still under zero strain, the strain resolution could again be determined. Table 1 outlines the strain resolution where the z-displaced scan has significantly poorer strain resolutions for all of the z-component strains. Given that the vector fields have 
been shown to be incorrect in this case, the strain resolution will be evaluated from the stationary and y-displaced scans. Previous studies have shown that any rigid body translation tests will cause an increase in strain standard deviation because of the additional errors from the interpolation biases due to the specimen movement [30], [26]. Although this is true for the z-displaced results, the y-displaced values are consistently lower than the stationary tests. It is unclear why this is the case, but there are several sources of mechanical or experimental errors that may have occurred. Table 1 shows that the $\varepsilon_{\mathrm{xx}}$ component, which describes the tensile opening strains across the interlayer, has a resolution of about $0.047 \%$, with the maximum value of $0.069 \%$ coming from the $\varepsilon_{y y}$ component in the stationary tests. Therefore, strain values obtained in the crack opening study that are above these values will be due to material deformation and not due to noise or other artefacts. However, the inability of the technique to track displacements along the fibre direction means that any z-component strains should be treated with caution and cannot be confidently regarded to be a true representation of the material behaviour.
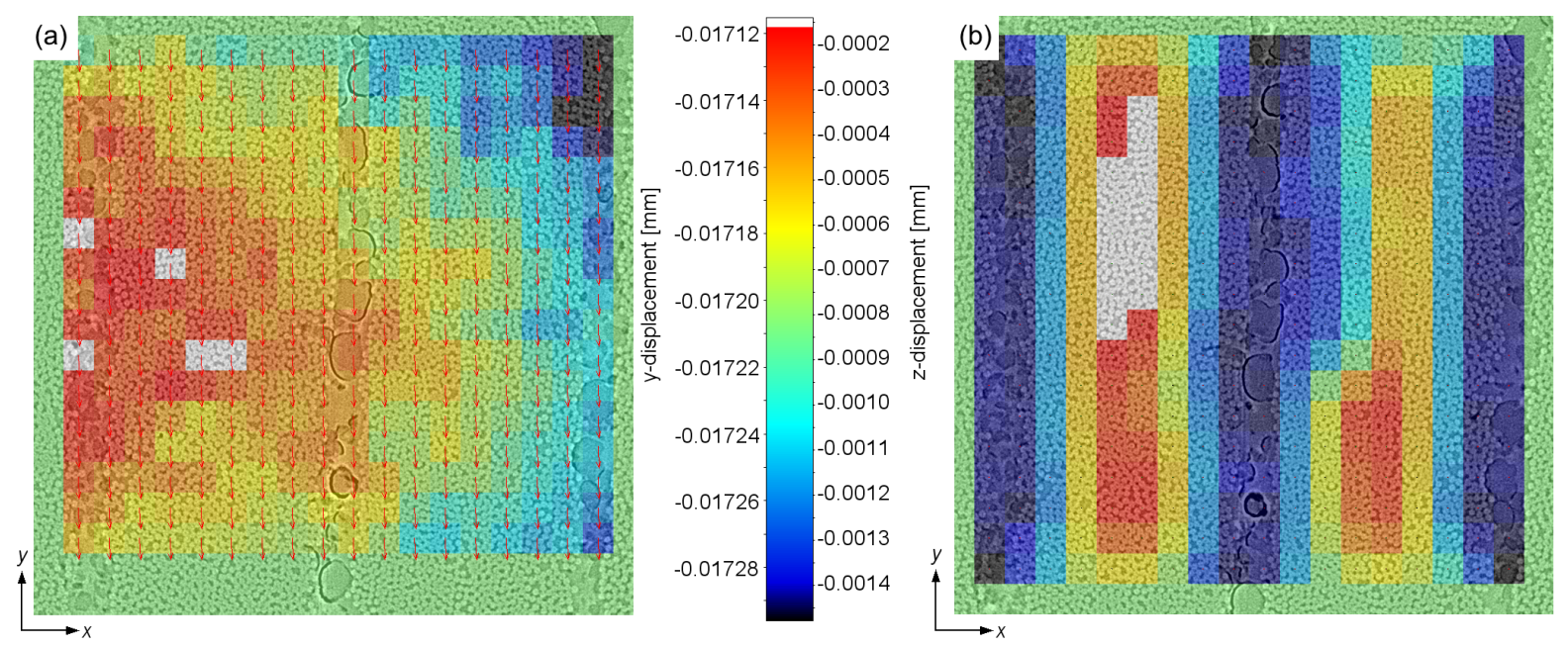

Figure 5 - Illustration of the displacement maps following an experimentally induced (a) $16.9 \mu \mathrm{m}$ y-translation, and (b) $-1.3 \mu \mathrm{m}$ z-translation 
Table 1 - Strain resolutions using a 150 voxel sub-volume size in stationary and rigid body translated scans

\begin{tabular}{ccccccc}
\multicolumn{7}{c}{ Strain Resolution [\%] } \\
& $\varepsilon_{\mathrm{xx}}$ & $\varepsilon_{\mathrm{xy}}$ & $\varepsilon_{\mathrm{xz}}$ & $\varepsilon_{\mathrm{yy}}$ & $\varepsilon_{\mathrm{yz}}$ & $\varepsilon_{\mathrm{zz}}$ \\
\hline Stationary & 0.0473 & 0.0400 & 0.0465 & 0.0691 & 0.0312 & 0.0215 \\
Y-displaced & 0.0282 & 0.0242 & 0.0150 & 0.0461 & 0.0193 & 0.0146 \\
Z-displaced & 0.0464 & 0.0418 & 0.6380 & 0.0691 & 0.1360 & 0.1750
\end{tabular}

\section{4 - Interlaminar strain measurements}

The strain values presented in the following section limited to a cut off value of 0.03 (or 3\%) because at this point the correlation coefficient drops below 0.8 (maximum of 1). In this work, this value reduces where there is a presence of new crack surfaces, which is caused during crack growth between load steps. It is acknowledged that there can be no strains where there is no material (i.e. a crack or de-bond), so the strain values quoted in this work refer to the average sub-volume strain, calculated from the relative displacement of the centroids of the neighbouring sub-volumes. Figures 6 and 7 show a number of CT slices through the materials with the image on top indicating the 'reference' or pre-growth crack position/microstructure, and the corresponding post-growth slice below it with the corresponding $\varepsilon_{\mathrm{xx}}$ strain map. The crack direction is from left to right. The vertical lines in the 'crack growth' images are separated by 75 pixels, illustrating the spacing between the neighbouring sub-volume centroids.

\section{1 - Damage evolution and fracture micro-mechanisms}

Figure 6(a) shows the CT slices and corresponding tensile/opening strain measurements for a slice within the volume of Mat. A. It is important to note that the average strain between the neighbouring sub-volume centroids is being presented. The process zone of Mode I crack growth is distributed, with the individual particles de-bonding ahead of the crack tip, followed by crack coalescence and the formation of bridging ligaments in the crack wake. These ligaments are formed from the bulk resin between the de-bonded particles (seen at 6(a)(i)), with larger ligaments formed (less frequently) from a mixture of resin and particle(s). It is believed that the main contributor to the toughness comes from the deformation of the bulk epoxy rather than the particles since they de-bond at too early a stage 
to carry load effectively across the crack faces. 3D investigation of the complete $\varepsilon_{\mathrm{xx}}$ strain field has shown that the particles de-bond at about 1-1.5\% strain, with significant additional damage including resin cracking, occurring above about $3 \%$. By having particles de-bonding ahead of the crack tip, the system facilitates a lower energy crack path, whereby the crack will follow the (de-bonded) particles rather than deviating to the interface, as has been seen to occur in similar interlayered systems [31].

Figure 6(b) shows the corresponding images for Mat. B. In this system, the propagation mechanism initiates from the particles cracking from within (6(b)(i)). After damage initiation, neighbouring cracks coalesce and leave behind bridging ligaments formed from the particles themselves (6(b)(ii)). Volumetric analysis of the $\varepsilon_{\mathrm{xx}}$ strain data has shown that these internal cracks appear at about $0.5-0.7 \%$ strain, with little evidence of any particle de-bonding events until above $2.0 \%$. From this, it can be deduced that these particles are better bonded to the surrounding resin that the particles in Mat. A. Additionally, particles that end up bridging the crack remain bonded to the crack faces, even as the thermoplastic necks at higher strain levels, shown at 6(b)(ii). This suggests that the effectiveness of the particlebridging mechanism is dependent upon the interface strength between the particles and the surrounding resin, and the ability for the particles to carry load effectively across the crack flanks by deforming plastically.

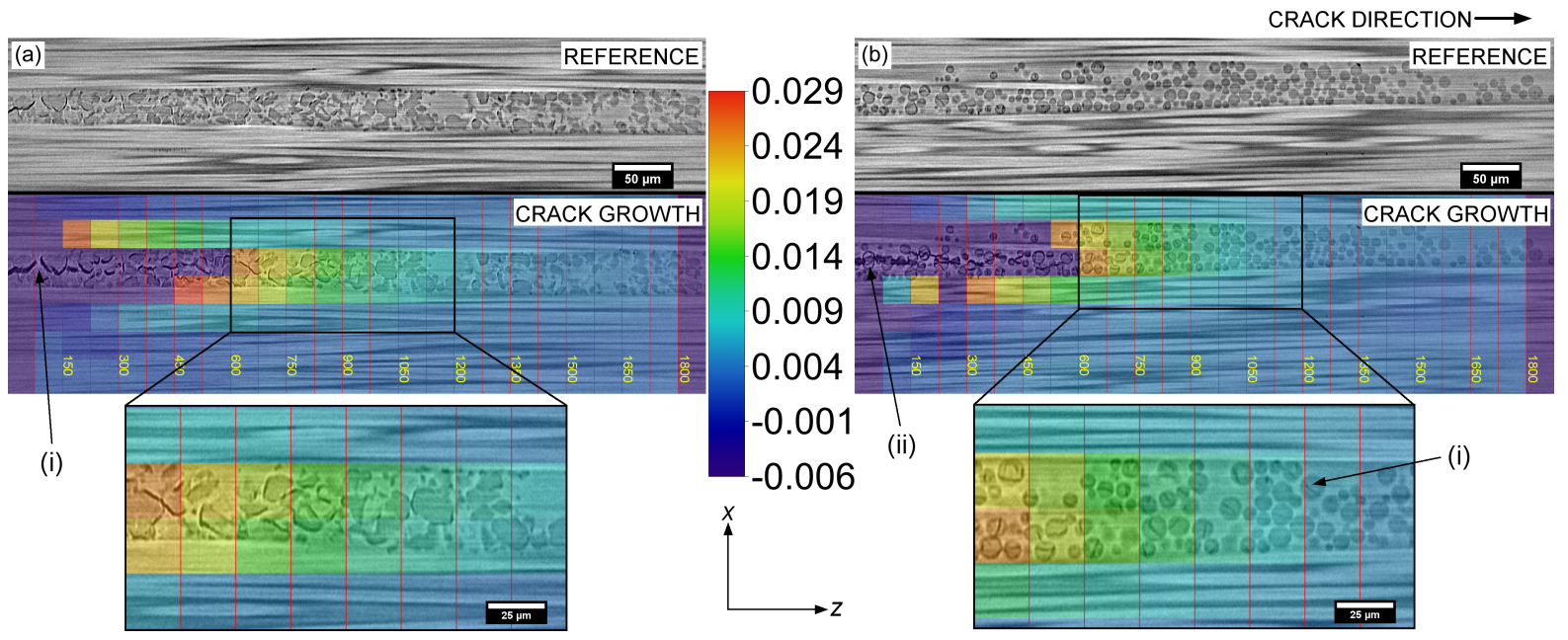

Figure 6-Showing reference and crack growth CT slices with the $\varepsilon_{\mathrm{xx}}$ strains overlaid for (a)

Mat. A, and (b) Mat. B 
Figures 7(a) and (b) show two different failure mechanisms occurring in Mat. C. It is clear from the highly divided microstructure that the Mat. A. particles are distributed along the top of the interlayer (7(a)(i)), with the other particles distributed along the bottom interface (7(a)(ii)). Previously published work has shown that Mode I crack propagation in a laminate containing solely the second particle type (not Mat A) occurred via intralaminar failure [22], with no evidence of particle de-bonding. Figure 7(a) shows the crack following the de-bonded Mat. A particles, with the first evidence of particle de-bonds occurring between 0.5-0.9\% strain, which is less than observed for a pure Mat. A laminate. It is suspected that because there are fewer de-bonds occurring ahead of the crack tip, that the competition between the lowest energy crack paths is much closer between the intralaminar and interlaminar route, which is why the some failure is seen within the ply. This intralaminar failure is seen in Figure 7(b), where although the particles are still de-bonding within the interlayer, the crack propagates within the ply (7(b)(i)), with the fibre bridging occurring in further the crack wake. Within the scanned volume, intralaminar failure occurred for the first $12 \%$ of the volume across the width of the sample. However, it is highly likely that this intralaminar failure extended beyond the field of view, which only captured a $0.7 \times 0.7 \times 0.7$ $\mathrm{mm}$ field of view at the centre of the specimen.
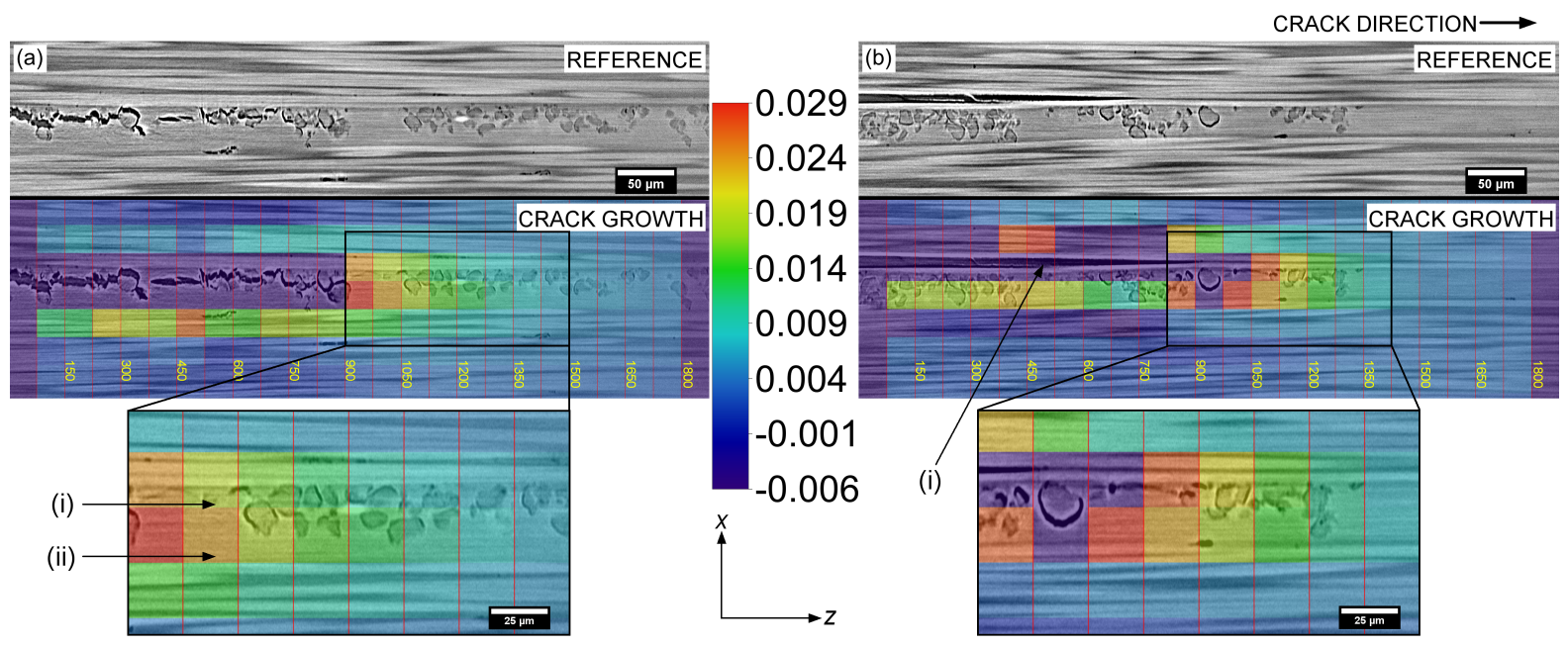

Figure 7 - Showing reference and crack growth CT slices with the $\varepsilon_{\mathrm{xx}}$ strains overlaid for Mat. C; featuring (c) interlaminar and (d) intralaminar failure

\section{2 -Interlayer strain profiles ahead of the crack tip}

Figure 8 illustrates the average $\varepsilon_{\mathrm{xx}}$ strain across the interlayer ahead of the crack tip, with the crack direction going from left to right. The average strain we define as a measure of 
the total strain across the interlayer, which is a measurement that can be compared to FE models from the change in displacement between two nodes on opposite sides of the interlayer. These values were calculated by taking the average of five sub-volume measurements vertically across the interlayer, for the entire scan volume. These are plotted at different positions along the length of the crack to show the $\varepsilon_{\mathrm{xx}}$ strain profile ahead of the crack tip. The strains are plotted up to $2 \%$, because beyond this point, the calculations often contained sub-volumes with a strain measurement above $3 \%$. It was deduced that at this point, that the correlation falls too low and reduces confidence in the measurements. Nonetheless, the plot shows that Mat. A. and Mat. B have a more gradual increase in strain opening, with the greatest increase in gradient in the magnified region measured in Mat. C. This is consistent with the micromechanical observations, that show that this material fails in a different manner featuring both inter- and intralaminar failure, where the intralaminar failure shows less distributed damage ahead of the crack tip. The longer micro-mechanical process zone ahead of the tip in Mat. A and Mat. B supports the more gradual increase in opening strain. These profiles may reflect the effectiveness of the bridging mechanisms, where other authors have reported a reduction in toughness once the crack migrates to the ply interface [31]. Such 3D strain data could be compared to FE Models, and in conjunction with crack opening displacement measurements behind the crack tip, can provide substantial insight into the behaviour both ahead and behind of the crack tip.

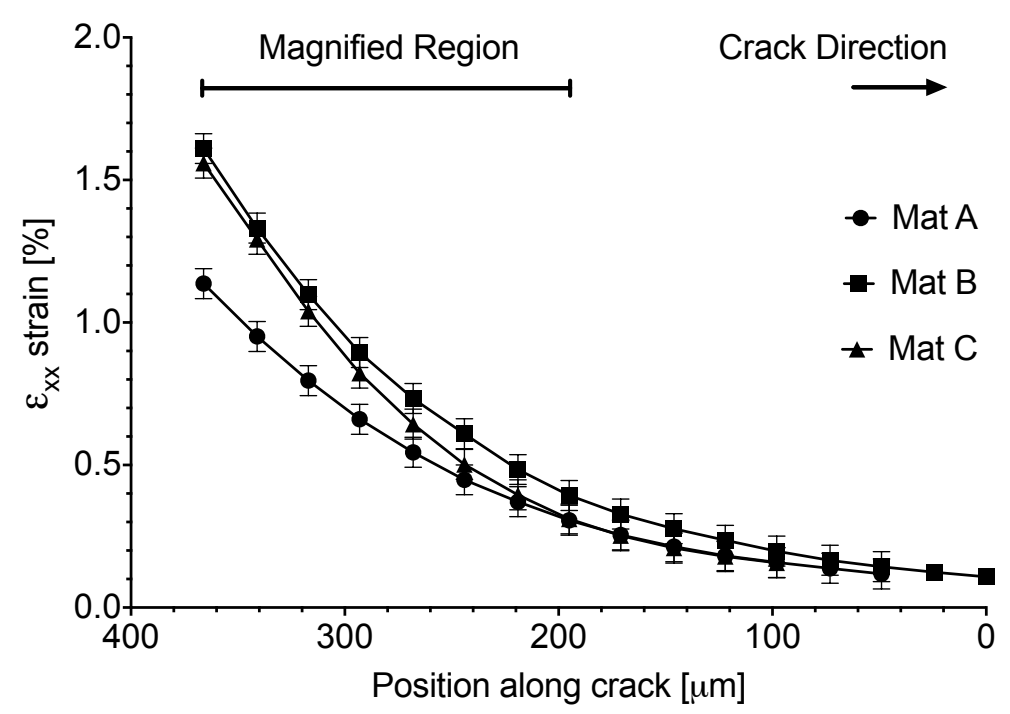

Figure $8-\varepsilon_{\mathrm{xx}}$ strain opening profile across the interlayer ahead of the crack tip, with the crack direction from left to right and the magnified region corresponding to Figures 6 and 7 


\section{$4.3-3 D$ analysis identifying strains neighbouring interlayers and in novel orientations}

Figure 9 shows a plan view of the interlayer microstructure overlaid with the corresponding $\varepsilon_{* *}$ strain measurements in Mat. A. All of the strains shown are above the noise threshold (between $0.1-3.0 \%$ ), with measurements on the left hand side of the interlayer removed due to a drop in the correlation coefficient. The figure identifies particle-depleted regions within the interlayer, highlighting the variation in micro-structure of this material system. The effects of such microstructural variations have been previously identified in other works [22], whereby such regions tend to promote brittle behaviour rather than the progressive evolution of damage experienced in particle-rich regions. The figure shows the number of de-bonded particles increasing from right-to-left, and a relatively uniform strain distribution across the width of the sample with perhaps a little more crack progression in the centre of the sample. This suggests that preliminary use of 2D models to model interlayer crack propagation may be adequate, given that significant alterations in opening strain profiles are not observed with respect to the length of the interlayer thickness. It is clear in these images, that there is much more that can be obtained by using this DVC technique, which can produce strain maps in the through-thickness and at orientations that are not possible with DIC.

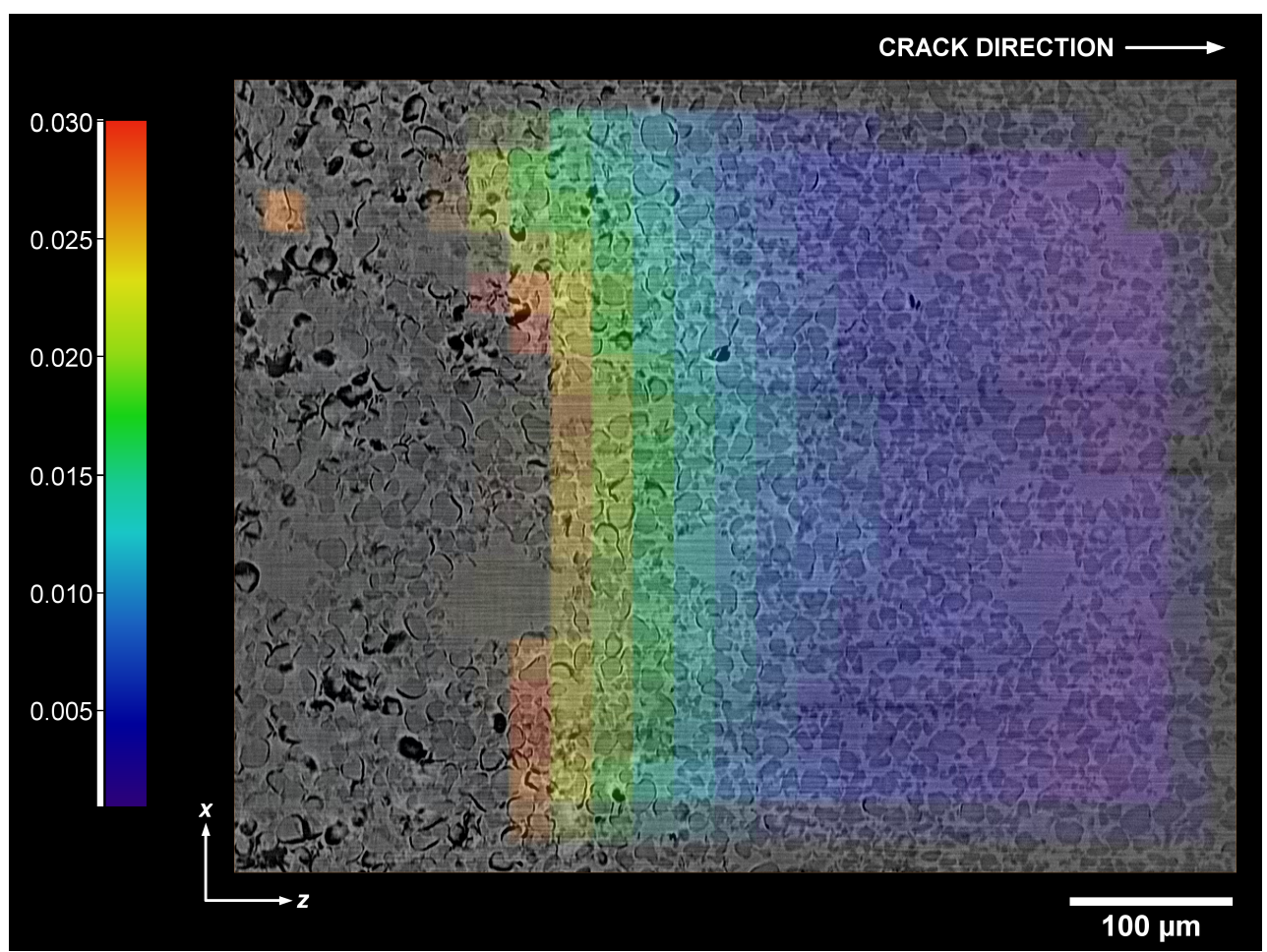

Figure 9 - Showing a plan view of the interlayer microstructure overlaid with corresponding $\varepsilon_{\mathrm{xx}}$ strains 


\section{5 - Conclusion}

The results presented in this work are targeted at increasing the understanding surrounding key mechanisms contributing to the toughness of interlayer-toughened systems. These time-series experiments are the first to capture Mode I crack growth in these material systems at such high resolutions, thus removing any doubt surrounding whether particles are reacting to the applied loads, or whether they were previously cracked/de-bonded. Additionally, estimates of the strain at which the different particles cracked and/or de-bonded were made; this could be used to inform future modelling work. Through the use of SRCT with DVC, a significant difference in opening strain curvature was identified for Mat. C, which featured a combination of inter- and intra-laminar failure. Two different bridging mechanisms were identified, with particle-bridges in Mat. B, and predominantly resin ligaments present in Mat. A. The observations on all of the material systems presented in this work emphasize that crack propagation in particle-toughened CFRPs is heterogeneous process, with distributed damage initiation sites, rather than due to the propagation of a single crack tip.

The DVC technique explored in this work has shown that strains ahead of the crack tip can be identified effectively, with the corresponding strain opening profiles suitable for future model validation. The limitations regarding the ability for the displacements to be tracked along the fibre directions has been demonstrated previously, but this work has shown that toughening particles are effective markers that can be tracked within the interlayers without disrupting the material micro-structure with other fiducial markers. The presence of these enabled the pre- and post-growth volumes to be aligned along the fibre direction, allowing measurements across the interlayer to be made with greater confidence.

\section{Acknowledgements}

The authors acknowledge contributions from institutions and staff: Cytec Industries Ltd. for their sponsorship and materials supply, and the support from Dr. Kingsley Ho as the technical point of contact. The $\mu$-VIS centre at the University of Southampton for provision of tomographic imaging facilities, supported by EPSRC grant EP-H01506X, and the support from Dr. Richard Boardman and Dr. Neil O'Brien. Additionally, the authors acknowledge support from Dr. Peter Modregger at the Swiss Light Source and funding from the Community's Seventh Framework Programme (FP7/2007-2013) under grant agreement n. ${ }^{\circ} 312284$ (for CALIPSO). 


\section{Bibliography}

[1] M.O.W. Richardson, M.J. Wisheart, Review of low-velocity impact properties of composite materials, Compos. Part A, 27 (1996) 1123-1131.

[2] M.R. Groleau, Y.-B. Shi, A.F. Yee, J.L. Bertram, H.J. Sue, P.C. Yang, Mode II fracture of composites interlayered with nylon particles, Compos. Sci. Technol., 56 (1996) 1223-1240.

[3] M. Yasaee, I.P. Bond, R.S. Trask, E.S. Greenhalgh, Mode I interfacial toughening through discontinuous interleaves for damage suppression and control, Compos. Part A, 43 (2012) 198-207.

[4] D.J. Bull, A.E. Scott, S.M. Spearing, I. Sinclair, The influence of toughening-particles in CFRPs on low velocity impact damage resistance performance, Compos. Part A, 58, (2014) 47-55.

[5] A.E. Scott, M.N. Mavrogordato, P. Wright, I. Sinclair, S.M. Spearing, In situ fibre fracture measurement in carbon-epoxy laminates using high-resolution computed tomography, Compos. Sci. Technol., 71 (2011) 1471-1477.

[6] S.C. Garcea, I. Sinclair, S.M. Spearing, In situ synchrotron tomographic evaluation of the effect of toughening strategies on fatigue micromechanisms in carbon fibre reinforced polymers, Compos. Sci. Technol., 109 (2015) 32-39.

[7] F. Gillard, R. Boardman, M. Mavrogordato, D. Hollis, I. Sinclair, The application of digital volume correlation (DVC) to study the microstructural behaviour of trabecular bone during compression, J. Mech. Behav. Biomed. Mater., 29 (2014) 480-499.

[8] B.K. Bay, S.A. Yerby, R.F. McLain, E. Toh, Measurement of strain distributions within vertebral body sections by texture correlation, Spine, 24 (1999) 10-17.

[9] A.D. Krawitz, Neutron strain measurement, Mater. Sci. Technol., 27 (2011) 589-603.

[10] B.K. Bay, T.S. Smith, D.P. Fyhrie, M. Saad, Digital volume correlation: threedimensional strain mapping using X-ray computed tomography, Experimental Mechanics, 39 (1999) 217-226.

[11] B.K. Bay, Methods and applications of digital volume correlation, J. Strain Anal. Eng. Des., 43 (2008) 745-760.

[12] M. Grediac, F. Pierron, S. Avril, E. Toussaint, The virtual fields method for extracting constitutive parameters from full-field measurements: a review, Strain, 42 (2006) 233-253.

[13] R. Brault, A. Germaneau, J. C. Dupre, P. Doumalin, S. Mistou, A. M. Fazzini, In-situ analysis of laminated composite materials by X-ray micro-computed tomography and digital volume correlation, Experimental mechanics, 53 (2013) 1143-1151.

[14] E.P. Durand, P. Ruegsegger, High-contrast resolution of CT images for bone-structure 
analysis, Medical Physics, 19 (1992) 569-573.

[15] R.P. Elke, E.J. Cheal, C. Simmons, R. Poss, Three-dimensional anatomy of the cancellous structures within the proximal femur from computed-tomography data, J. Orthop. Res., 13 (1995) 513-523.

[16] D.J. Bull, L. Helfen, I. Sinclair, S.M. Spearing, T. Baumbach, A comparison of multiscale 3D X-ray tomographic inspection techniques for assessing carbon fibre composite impact damage, Compos. Sci. Technol., 75 (2013) 55-61.

[17] T.S. Smith, B.K. Bay, M.M. Rashid, Digital volume correlation including rotational degrees of freedom during minimization, Experimental Mechanics, 42 (2002) 272-278.

[18] S. Hall, M. Bornert, J. Desrues, Y. Pannier, N. Lenoir, G. Viggiani, P. Besuelle, Discrete and continuum analysis of localised deformation in sand using X-ray mu-CT and volumetric digital image correlation, Geotechnique, 60 (2010) 315-322.

[19] S. Roux, F. Hild, P. Viot, D. Bernard, Three-dimensional image correlation from X-ray computed tomography of solid foam, Compos. Part A, 39 (2008) 1253-1265.

[20] F. Forsberg, C.R. Saviour, 3D deformation and strain analysis in compacted sugar using X-ray microtomography and digital volume correlation, Meas. Sci. Technol, 20 (2009) 095703.

[21] S.M. Spearing, A.G. Evans, The role of fibre bridging in the delamination resistance of fibre-reinforced composites, Acta Metall. et Mater., 40 (1992) 2191-2199.

[22] G. Borstnar, M.N Mavrogordato, L. Helfen, I. Sinclair, S.M. Spearing, Interlaminar fracture micro-mechanisms in toughened carbon fibre reinforced plastics investigated via synchrotron radiation computed tomography and laminography, Compos. Part A, 71 (2015) 176-183.

[23] F. Marone, M. Stampanoni, Regridding reconstruction algorithm for real-time tomographic imaging, J. Synchrotron Radiat., 19 (2012) 1029-1037.

[24] LaVision, DaVis 3.1.3 software, Goettingen, Germany.

[25] MATLAB version 7.10.0. Natick, Massachusetts: The MathWorks Inc., 2010.

[26] F. Pierron, S.A. McDonald, D. Hollis, J. Fu, P.J. Withers, A. Alderson, Comparison of the mechanical behaviour of standard and auxetic foams by X-ray computed tomography and digital volume correlation, Strain, 49 (2013) 467-482.

[27] L. Liu, E.F. Morgan, Accuracy and precision of digital volume correlation in quantifying displacements and strains in trabecular bone, J. Biomech., 40 (2007) 3516-3520.

[28] M.A. Sutton, C. Mingqi, W.H. Peters, Y.J. Chao, S.R. McNeill, Application of an optimized digital correlation method to planar deformation analysis, Image and Vision 
Comput., 4 (1986) 143-150.

[29] S. Yaofeng, J.H.L. Pang, Study of optimal subset size in digital image correlation of speckle pattern images, Opt. Laser Eng., 45 (2007) 967-974.

[30] K. Madi, G. Tozzi, Q.H. Zhang, J. Tong, A. Cossey, A. Au, D. Hollis, F. Hild, Computation of full-field displacements in a scaffold implant using digital volume correlation and finite element analysis, Med. Eng. Phys., 35 (2013) 1298-1312.

[31] M. Hojo, S. Matsuda, M. Tanaka, S. Ochiai, A. Murakami, Mode I delamination fatigue properties of interlayer-toughened CF/epoxy laminates, Compos. Sci. Technol., 66 (2006) 665-675. 